\title{
Clinical advances in nasopharyngeal carcinoma surgery and a video demonstration
}

\author{
Xi Ding ${ }^{1,2, \#}$, You-Ping Liu ${ }^{1,2, \#}$, Yi-Jun Hua ${ }^{1,2}$, Xiong Zou ${ }^{1,2}$, Zhi-Qiang Wang ${ }^{1,2}$, \\ Yu-Long Xie ${ }^{1,2}$, and Ming-Yuan Chen ${ }^{1,2, *}$ \\ ${ }^{1}$ Department of Nasopharyngeal Carcinoma, Sun Yat-sen University Cancer Center, 651 Dongfeng East Road, \\ Guangzhou 510060, P. R. China \\ 2 Sun Yat-Sen University Cancer Center; State Key Laboratory of Oncology in South China Collaborative Innovation Center for Cancer \\ Medicine; Guangdong Key Laboratory of Nasopharyngeal Carcinoma Diagnosis and Therapy, Guangzhou 510060, China
}

Received 19 August 2020, Accepted 25 December 2020, Published online 27 April 2021

\begin{abstract}
Nasopharyngeal carcinoma (NPC) is highly radiosensitive, and radiotherapy is recommended for newly diagnosed NPC. Because of the poor visual surgical field, narrow operating space, difficulty protecting the internal carotid artery (ICA) and poor wound healing, the development of NPC surgery has been severely limited. For recurrent NPC, some open surgical approaches, such as the maxillary swing, successfully solve the above major problems. However, these operations are traumatic and lead to many postoperative complications. With the development of minimally invasive surgery, two concepts, the "third-hand technique" and "dumpling making technique", have been proposed, combining with the intraoperative navigation systems and multiple anatomical landmarks for identifying ICA. Endoscopic nasopharyngectomy (ENPG) can also break through the above restrictions and has become a first-line treatment for locally recurrent NPC. Moreover, a new surgical staging system for recurrent NPC was devised to aid clinicians in choosing the most suitable treatment for these patients. A current study on ENPG alone for newly diagnosed stage I NPC shows that the long-term survival outcomes after ENPG are similar to those after IMRT. ENPG was associated with low medical costs and satisfactory QOL and might be an alternative strategy for treating newly diagnosed localized stage I NPC patients who refuse radiotherapy.
\end{abstract}

Key words: Nasopharyngeal carcinoma, Recurrent, Surgery, Endoscopic nasopharyngectomy

\section{Introduction}

Nasopharyngeal carcinoma (NPC) has an obvious geographical pattern of incidence and is endemic in Southern China and Southeast Asia with an annual incidence of 10-40/100,000 [1]. Radiotherapy is the first choice for newly diagnosed NPC. However, surgery, the classical treatment to cure malignant tumors, has not been recommended for newly diagnosed NPC for the following reasons: (1) undifferentiated nonkeratinizing squamous cell carcinoma (NKSCC) accounts for approximately $95 \%$ of Chinese NPC patients. NKSCC is highly malignant-prone to infiltration into the skull base and metastasis to the cervical lymph nodes-making it difficult to achieve radical resection of the nasopharyngeal primary tumors, cervical lymph nodes, and communicating lymphatic vessels. However, this tumor is highly radiosensitive, and radiation exposure can cover the above lesions simultaneously. The efficacy of radiotherapy can be further improved after combined chemotherapy.

*Corresponding author: chmingy@mail.sysu.edu.cn

\# Ding Xi, You-Ping Liu contributed equally to this study.
The 5-year OS rates of stage I-IV patients were $96.3 \%-100 \%$, $91.2 \%-95.1 \%, 87.5 \%-88.3 \%$, and $78.6 \%-80 \%$, respectively [2-5]. (2) The nasopharynx is located in the center of the skull base, and the surrounding tissues and organs do not move, which makes it possible to position for radiotherapy repeatably and precisely, but inaccessible to resect for surgery. Furthermore, the surrounding radiosensitive structures can tolerate radiation well and are suitable for high-dose radiotherapy. (3) Intensity-modulated radiotherapy (IMRT) can achieve excellent local control with a high dose of radiation to the tumors and results in few radiotherapy complications by limiting the dose to normal tissues. In addition, IMRT has been shown to reduce long-term toxicity in the salivary glands, temporal lobes, connective tissue, and auditory and optic structures [6] and has become the first choice for newly diagnosed NPC.

Approximately $10-36 \%$ of patients relapse after radiotherapy. Recurrent lesions are radiation-resistant; thus, the efficacy of reirradiation is poor, and the 5-year Overall Survival (OS) rate of recurrent NPC is only $26 \%-45 \%$ [7-9]. Furthermore, the radiation-related complications were severe, and the 
incidence of late radiation-induced injury above grade III in rT1-2 and rT3-4 patients was $45.8 \%$ and $78.3 \%$, respectively. Moreover, $28.5 \%$ and $40.6 \%$ of patients developed new radiation encephalopathy and nasopharyngeal necrosis, respectively, which seriously affected the patient quality of life [8].

Compared with salvage IMRT, salvage surgery has obvious advantages for locally recurrent NPC: surgery can directly remove lesions insensitive to radiotherapy while avoiding reirradiation damage, and the related complications are relatively mild. In addition to killing the visible primary tumor and metastatic lymph nodes, the first cycle of radiotherapy also seals the lymph channels. Therefore, for recurrent NPC or regional lymph nodes, only the isolated lesions need radical resection without the need for radical surgery for primary nasopharyngeal lesions, neck lymph nodes, and communications with lymphatic vessels. However, in clinical practice, more recurrent NPCs are treated with reirradiation rather than salvage surgery. According to the NCCN guidelines (version 1.2019), surgery is not the only choice even for "resectable" recurrent NPC, and systemic therapy or radiotherapy is also recommended. The root cause is that traditional nasopharyngectomy is very invasive, difficult to perform, and not widely popular. The majority of traditional open surgical approaches require a facial incision to expose the nasopharyngeal cavity. The nasopharynx is adjacent to many important organs, especially the internal carotid artery (ICA) located in the parapharyngeal space, which can lead to fatal hemorrhage if accidentally injured. Therefore, only a few cancer centers can carry out such operations, while most patients with local recurrence still need to receive reirradiation.

The development of NPC surgery technology has faced three major problems: (1) the limited visual field and narrow operation space make it difficult to achieve radical resection; (2) recognition of the ICA is not easy, and the consequences of accidental injury are serious; and (3) the postoperative nasopharyngeal incision heals poorly, which affects patient quality of life.

\section{Salvage surgery for locally recurrent nasopharyngeal cancer}

\section{Traditional open surgery}

Access to the nasopharynx varies with different directions, such as the anterior approach (the midfacial degloving procedure), anterior-lateral approach (maxillary swing), lateral approach (infratemporal fossa approach and trans-mandibularpterygoid approach), inferior approach (transpalatal approach), and transcervical approach with widespread exposure. Among these approaches, the maxillary swing successfully solved the above three major problems [10]: First, it provides complete exposure of the ipsilateral nasopharynx down to the oropharynx inferiorly and the parapharyngeal space posterolaterally. With the removal of the posterior part of the nasal septum, the contralateral nasopharynx can also be exposed. Safe resection of tumors invading the parapharyngeal space and those with retropharyngeal lymph node metastasis can be performed. Second, the ICA can be identified and safeguarded by intraoperative palpation, ultrasound, or CT navigation. Finally, the maxillary osteo-cutaneous flap can maintain the blood supply when swung out. The temporalis muscle flap or microvascular free flap can be employed to protect exposed arteries to avoid blow-out bleeding; thus, the incidence of postoperative maxillary osteonecrosis and massive hemorrhage is low $(0-1 \%)$, and wound healing occurs more easily than with the other approaches $[11,12]$. Therefore, the maxillary swing has become one of the most influential open surgical approaches with the largest number of reported cases. In the largest series involving 338 patients (72\% rT1-2) who received the maxillary swing for recurrent NPC, $78.4 \%$ of patients had microscopically clear resection margins, and the 5-year disease-free survival (DFS) rate was $63 \%$ for patients with negative margins and $37 \%$ for those with positive margins. However, traumatic resection can lead to a higher incidence of long-term procedure-related complications. Overall, 40.8\% of patients developed middle ear effusion, and 11.2\%, 10.9\%, $9.5 \%$ and $7.1 \%$ had trismus, palatal fistula, osteoradionecrosis, and facial numbness, respectively [11].

\section{Endoscopic nasopharyngectomy (ENPG)}

In the late 1990s, with the development of nasal endoscopic instruments and the maturation of functional endoscopic surgical techniques, minimally invasive surgery applied for the treatment of sinonasal malignancies began to emerge. In the early 2000s, the application of ENPG for locally recurrent NPC also started and gradually became mainstream. The first reported article on ENPG for recurrent NPC was reported by MY Chen in 2007 [13]. Among 25 patients (rT1-3), only 1 had a positive surgical margin, and no patients received postoperative radiotherapy (the patient with a positive margin refused it). The 1-year OS and local recurrence-free survival (LRFS) rates were $100 \%$ and $86 \%$, respectively. No surgical complications or perioperative deaths occurred. In 2009, the authors once again reported 37 patients, including 17, 4, 14, and 2 patients staged as rT1NOM0, rT2aNOM0, rT2bNOM0, and rT3NOM0, respectively. Thirty-five patients achieved en bloc tumor resection with negative surgical margins. In the remaining two cases, the tumors were dissected into pieces, and one patient showed positive surgical margins. No patients received postoperative radiotherapy (the positive-margin patient refused), and no severe complications were observed. The 2-year OS, LRFS, and progression-free survival (PFS) rates were $84.2 \%, 86.3 \%$, and $82.6 \%$, respectively [14]. These two studies have shown that nasal endoscopic salvage surgery has good prospects for early recurrent NPC.

Moreover, MY Chen and his colleagues proposed eligible solutions to the major problems: (1) Exposure of the nasopharynx and radical resection: the nasopharynx is basically surrounded by bone. In endoscopic surgery, as long as dissection and excision occur along the bone surface, the mucosa of the anterior wall, superior wall, and posterosuperior wall of the nasopharynx can be removed. Furthermore, the "third-hand technique" was promoted to allow an assistant to use one or two straws to pull tissues and aspirate blood and smoke through the bilateral nasal cavities to reduce the inconveniences of the operation. The "dumpling making technique" was developed 
to wrap the tumor using surrounding normal nasopharyngeal mucosae so that the surgical instrument could not contact the tumor directly, and the nasopharyngeal tumors could be removed en bloc, rather than in pieces, based on the principles of no-touch and en bloc resection. (2) Wound healing: wound recovery is necessary for NPC patients receiving postoperative radiotherapy. The pedicled middle turbinate mucoperiosteal flap was devised by MY Chen in 2007, and the study showed that $96 \%$ of patients achieved complete wound closure [13]. To expand the repair area further, a pedicle nasal septum and floor mucoperiosteal flap was reported among 12 locally recurrent NPC patients, with 2 patients receiving 2 cycles of radiotherapy. Finally, all flaps successfully covered all nasopharyngeal defects and relined the nasopharynx with good functional recovery [15]. (3) Protection of the ICA: because of the inability to expose and directly visualize the ICA during nasal endoscopic surgery, blow-out of ICA has been regarded as the biggest constraint for ENPG. Therefore, MY Chen and his colleagues strictly limited the indications and chose tumors that were $\geq 0.5 \mathrm{~cm}$ away from the ICA to ensure en bloc resection without damaging it. At present, multiple anatomical landmarks can be applied to comprehensively locate the ICA, such as the deep part of the eustachian tube, the tensor veli palatini muscle, and canalis pterygoideus [16-18]. Coupled with intraoperative navigation systems and ultrasound positioning, the safety of the ICA can be guaranteed.

Video 1 is a real example demonstrating the ENPG procedure. The patient was diagnosed with recurrent NPC, staged as rT2NOM0 and overall recurrent stage II. Since the tumor was more than $0.5 \mathrm{~cm}$ away from the ICA, the patient was treated with ENPG after excluding any surgical contraindications. The operation was performed with the endoscopic endonasal approach. Exploration revealed that the tumor was located in the right pharyngeal recess with a relatively clear margin. Before the operation, boundary marking was conducted, and the tumor was marked with an additional 5-10 mm surrounding normal tissue to ensure a safe margin. The anterior, superior, left and right lateral, inferior and posterior walls were excised in order. After the gross tumor was removed, a pedicle nasal septum and floor mucoperiosteal flap were used to reconstruct the nasopharyngeal defect. Three months later, the endoscopic image demonstrated that no abnormal neoplasm was observed in the nasopharynx, and the nasopharyngeal defect was completely re-epithelialized.

According to surgical practices for recurrent NPC patients, Chen et al. pioneered the specific definition of "resectable area" for ENPG in 2009 to include rNPC with tumors confined to the following locations: 1) the nasopharyngeal cavity (rT1); 2) the postnaris or nasal septum (rT2a); 3) the superficial parapharyngeal space (rT2b); or 4) the base wall of the sphenoid sinus (T3); in contrast, all rT4 diseases were unresectable by ENPG according to the 2002 clinical staging system of the UICC/ AJCC. To promote the application of ENPG as salvage nasopharyngectomy, MY Chen and his colleagues established a new surgical staging system for patients with recurrent NPC with the largest series $(n=894)$ based on the UICC/AJCC rTNM staging system [19]. In detail, all UICC/AJCC clinical recurrent T1-4 and recurrent N0-3 tumors were stratified as resectable and unresectable based on the imaging data of the

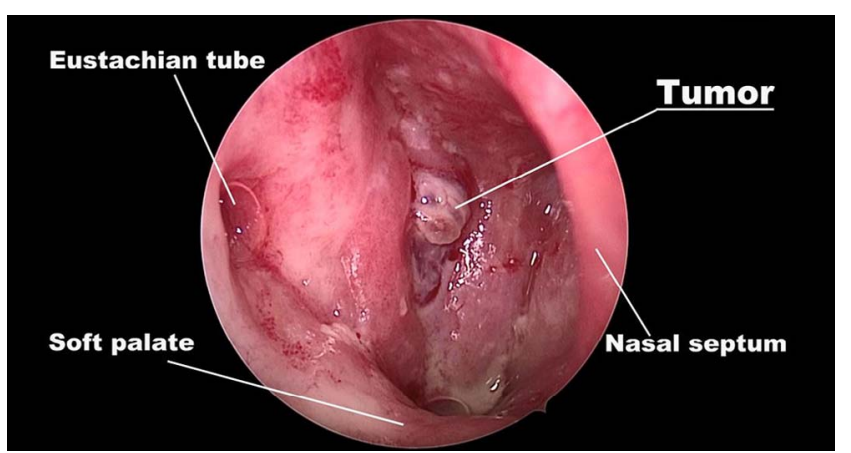

Video 1. Procedure of endoscopic nasopharyngectomy and reconstruction of nasopharyngeal mucosa for recurrent NPC. The video presents an endoscopic nasopharyngectomy, followed by resurfacing the nasopharyngeal with a pedicle nasal septum and floor mucoperiosteal flap for a 31-year-old man with rT2NOM0 and overall recurrent stage II (American Joint Committee on Cancer seventh edition classification) non-keratinizing squamous cell carcinoma of right nasopharynx at 2018. https://vcm.edpsciences.org/10.1051/vcm/2021001\#V1

head and neck according to the above definitions, and there were 8 subgroups in the rT stage and 7 subgroups in the $\mathrm{rN}$ stage. These stages were further subdivided into surgical $\mathrm{T}$ stages and surgical $\mathrm{N}$ stages with similar clinical characteristics and death risks and were reintegrated into 4 new "surgical" stages using a Cox proportional hazards model, which are minimal (stage I), limited (stage II), extensive (stage III), and disseminated recurrence (stage IV). For "surgical" stage I (sI) patients, ENPG was associated with a better OS than 2D-CRT and IMRT (5-year OS rates: 93.4\%, 26.8\%, and $71.1 \%$, respectively; $P<0.001$ ); therefore, ENPG is preferred. In "surgical" stage II, the 5-year OS rates of ENPG and IMRT were $61.8 \%$ and $53.8 \%(P=0.14)$, respectively. Although there was no statistical significance between the two treatments, ENPG was probably related to fewer complications and lower risk. In "surgical" stage III, the lesion is "unresectable", and IMRT was associated with a better OS than 2D-CRT and chemotherapy alone for sT3 patients (5-year OS rates: $27.7 \%$, $15.9 \%$, and $16.2 \%$, respectively; $P=0.022$ ); thus, IMRT is the first choice. In "surgical" stage IV, a combination of aggressive treatment (surgery or reirradiation) and palliative chemotherapy showed an improved 5-year OS compared with palliative chemotherapy alone and no treatment; however, the differences did not achieve statistical significance (OS rates: $16.4 \%, 8.1 \%$, and $0 \%$, respectively; $P=0.482$ ). In short, compared with the recurrent TNM staging system, the "surgical" staging system exhibited improved prognostic value for predicting recurrent NPC patient survival and could aid clinicians in choosing the most suitable treatment for recurrent NPC patients. Moreover, a well-balanced cohort of 144 limited recurrent NPC patients was created by matching each patient who underwent ENPG with one who received IMRT [20]. The results indicated that ENPG was associated with a relatively good 5-year OS rate (77.1\% vs. $55.5 \%, P=0.003$ ), QOL conservation (mean global health status score, 57.6 vs. $29.8, P<0.001$ ), and significant reduction in post-treatment complications $(12.5 \%$ vs. $65.3 \%$, $P<0.001)$ and medical costs compared with IMRT. The corresponding prospective study has been completed, and the results will be reported soon. 
Currently, the Chinese Expert consensus on the treatment of recurrent nasopharyngeal carcinoma notes that for patients with locally recurrent NPC, ENPG is more suitable for the resectable rT1-2 stage, and radiotherapy should be supplemented for patients with a positive margin. For the rT3-4 phase, precise radiotherapy is the main treatment. Moreover, surgery is the first choice for regionally recurrent NPC. The Nasopharyngeal carcinoma: United Kingdom National Multidisciplinary Guidelines note that surgery is the first choice for locally recurrent NPC, and radiotherapy is only used as a second-line treatment.

\section{Minimally invasive surgery alone for newly diagnosed NPC}

Considering that ENPG has been successfully promoted and applied in recurrent NPC, some early malignant tumors, such as early esophageal cancer, early gastric cancer, and early glottic cancer, can currently be cured by endoscopic surgery. In addition, recent studies have reported that early screening technology for NPC can find $17.3 \%-47 \%$ of patients with stage I disease at the time of first diagnosis [21, 22], which provides a good research background for endoscopic salvage surgery.

Under the premise of ensuring a similar efficacy to IMRT and not violating the principles of surgical treatment for malignant tumors, we reported 10 newly diagnosed localized stage I NPC patients who voluntarily underwent ENPG alone [23]. Simultaneously, the data of 329 stage I NPC patients treated with IMRT were collected and used as a reference cohort. The indication criteria for ENPG were very strict: (1) stage $\mathrm{T} 1$ with a maximum primary tumor diameter $\leq 1.5 \mathrm{~cm}$ for radical resection and to reduce local recurrence and distant metastasis; (2) distance from the tumor margin to the ICA was $\geq 0.5 \mathrm{~cm}$ to avoid accidental injury; and (3) the minimal axial diameter on magnetic resonance imaging (MRI) was no more than $0.4 \mathrm{~cm}$ for the retropharyngeal lymph nodes (RPLNs) and $0.6 \mathrm{~cm}$ for the cervical lymph node (CLNs) smaller than the radiographic diagnostic criteria for lymph node metastasis $(0.5 \mathrm{~cm}$ for RPLNs and $1 \mathrm{~cm}$ for CLNs). Based on the fact if the size of the nasopharyngeal tumor, RPLNs, and CLNs are less than the above strict thresholds, the risk of subclinical lesions before surgery and recurrence and metastasis after surgery will be significantly reduced [24-27]. Therefore, there is no need for auxiliary treatment such as preventive neck dissection or neck radiotherapy after surgery to achieve the effect of radical resection for early NPC. The results showed that the 5-year OS, LRFS, regional relapse-free survival, and distant metastasis-free survival rates of the ENPG-treated patients were similar to those of the IMRT-treated patients (100\% vs. $99.1 \%, 100 \%$ vs. $97.7 \%, 100 \%$ vs. $99.0 \%, 100 \%$ vs. $97.4 \%$, respectively, $P>0.05)$. In addition, compared with IMRT, ENPG was associated with reduced total medical costs and improved QOL scores, including for dry mouth, sticky saliva, pain, and swallowing.

Therefore, we think ENPG might be an alternative strategy for treating newly diagnosed localized stage I NPC patients who refuse radiotherapy. While it must be emphasized that this cohort has been strictly selected for, and it remains to be seen if the quality were repeatable by other centers of different levels. The contemporaneous review by Melvin et al. from the National Cancer Centre Singapore [28] stated that the results are impressive with regard to the possibility of replacing IMRT with ENPG in early-stage NPC patients without compromising the excellent prognosis, but the generalized application of ENPG for low-risk NPC should still be approached prudently, and prospective clinical trials are needed to further verify the results.

\section{Conclusion}

With deep insight into the biological characteristics of NPC and evolving treatment technologies, the treatment methods for NPC have been gradually enriched and updated. At present, radiotherapy is still the first choice for newly diagnosed NPC, and ENPG has become the first-line therapy for SI-II local recurrent NPC. Given the iterative upgrades of early screening technology, an increasing number of early patients can be found. By strictly restricting the indication criteria, this prudent radical surgical treatment for "very early" stage NPC is expected to become an alternative to radiotherapy for patients who are unwilling to experience radiotherapy toxicity. However, more studies and patients are needed in the future to validate these results.

Acknowledgements. Funding was provided by the Program of Sun Yat-Sen University for Clinical Research 5010 Program (No. 2013010, 2018015, 2018029), the National Natural Science Foundation of China (No. 81772895, 81874134), Guangdong Province Science and Technology Development Special Funds (Frontier and Key Technology Innovation Direction - Major Science and Technology Project) (No. 2017B020226004), CSCO-JunShi Cancer Immunotherapy Clinical Research Fund (No. Y-JS2019-002).

\section{Competing interests}

The authors declare that they have no conflicts of interest.

\section{References}

1. Cao SM, Simons MJ, Qian CN. The prevalence and prevention of nasopharyngeal carcinoma in China. Chin J Cancer. 2011;30(2): 114-9.

2. Chen C, Yi W, Gao J, et al. Alternative endpoints to the 5-year overall survival and locoregional control for nasopharyngeal carcinoma: A retrospective analysis of 2,450 patients. Mol Clin Oncol. 2014;2(3):385-92.

3. Sun X, Su S, Chen C, et al. Long-term outcomes of intensitymodulated radiotherapy for 868 patients with nasopharyngeal carcinoma: an analysis of survival and treatment toxicities. Radiotherapy and Oncology: Journal of the European Society for Therapeutic Radiology and Oncology. 2014;110(3):398-403.

4. Zhang M-X, Li J, Shen G-P, et al. Intensity-modulated radiotherapy prolongs the survival of patients with nasopharyngeal carcinoma compared with conventional two-dimensional radiotherapy: A 10-year experience with a large cohort and long follow-up (Oxford, England: 1990). Eur J Cancer. 2015;51(17): 2587-95. 
5. OuYang PY, Shi D, Sun R, et al. Effect of intensity-modulated radiotherapy versus two-dimensional conventional radiotherapy alone in nasopharyngeal carcinoma. Oncotarget. 2016;7(22): 33408-17.

6. Huang TL, Chien CY, Tsai WL, et al. Long-term late toxicities and quality of life for survivors of nasopharyngeal carcinoma treated with intensity-modulated radiotherapy versus nonintensity-modulated radiotherapy. Head Neck. 2016;38(Suppl 1): E1026-32.

7. Wei WI, Kwong DLW. Current management strategy of nasopharyngeal carcinoma. Clin Exp Otorhinolaryngol. 2010; $3(1): 1-12$.

8. Han F, Zhao C, Huang S-M, et al. Long-term outcomes and prognostic factors of re-irradiation for locally recurrent nasopharyngeal carcinoma using intensity-modulated radiotherapy. Clin Oncol. 2012;24(8):569-76.

9. Au KH, Ngan RKC, Ng AWY, et al. Treatment outcomes of nasopharyngeal carcinoma in modern era after intensity modulated radiotherapy (IMRT) in Hong Kong: A report of 3328 patients (-HKNPCSG 1301 study). Oral Oncol. 2018;77:16-21.

10. Chan JY, Wei WI. Critical appraisal of maxillary swing approach for nasopharyngeal carcinoma. Expert Opin Ther Targets. 2012;16(Suppl 1):S111-7.

11. Chan JY, Tsang RK, Wei WI. Morbidities after maxillary swing nasopharyngectomy for recurrent nasopharyngeal carcinoma. Head Neck. 2015;37(4):487-92.

12. Chan JY, Wong ST, Chan RC, et al. Extracranial/intracranial vascular bypass and craniofacial resection: New hope for patients with locally advanced recurrent nasopharyngeal carcinoma. Head Neck. 2016;38(Suppl 1):E1404-12.

13. Chen MY, Xiang G, Wen WP, et al. Salvage surgical operation via endoscopic transnasal approach for local persistent or recurrent nasopharyngeal carcinoma. Chin J Cancer. 2007;26(07):673-8.

14. Chen MY, Wen WP, Guo X, et al. Endoscopic nasopharyngectomy for locally recurrent nasopharyngeal carcinoma. Laryngoscope. 2009;119(3):516-22.

15. Chen MY, Wang SL, Zhu YL, et al. Use of a posterior pedicle nasal septum and floor mucoperiosteum flap to resurface the nasopharynx after endoscopic nasopharyngectomy for recurrent nasopharyngeal carcinoma. Head Neck. 2012;34(10):1383-8.

16. Liu J, Sun X, Liu Q, et al. Eustachian tube as a landmark to the internal carotid artery in endoscopic skull base surgery. Otolaryngology-Head and Neck Surgery: Official Journal of American Academy of Otolaryngology-Head and Neck Surgery. 2016;154(2):377-82.

17. Liu CL, Hsu NI, Shen PH. Endoscopic endonasal nasopharyngectomy: tensor veli palatine muscle as a landmark for the parapharyngeal internal carotid artery. Int Forum Alergy Rhinol. 2017;7(6):624-8.

18. Wang WH, Lieber S, Mathias RN, et al. The foramen lacerum: surgical anatomy and relevance for endoscopic endonasal approaches. J Neurosurg. 2018;1-12.

19. You R, Zou X, Wang SL, et al. New surgical staging system for patients with recurrent nasopharyngeal carcinoma based on the AJCC/UICC rTNM classification system. Eur J Cancer (Oxford, England: 1990). 2015;51(13):1771-9.

20. You R, Zou X, Hua YJ, et al. Salvage endoscopic nasopharyngectomy is superior to intensity-modulated radiation therapy for local recurrence of selected T1-T3 nasopharyngeal carcinoma A case-matched comparison. Radiotherapy and Oncology: Journal of the European Society for Therapeutic Radiology and Oncology. 2015;115(3):399-406.

21. Ji MF, Huang QH, Yu X, et al. Evaluation of plasma EpsteinBarr virus DNA load to distinguish nasopharyngeal carcinoma patients from healthy high-risk populations in Southern China. Cancer. 2014;120(9):1353-60.

22. Chan KCA, Woo JKS, King A, et al. Analysis of plasma Epstein-Barr virus DNA to screen for nasopharyngeal cancer. New Engl J Med. 2017;377(6):513-22.

23. Liu YP, Lv X, Zou X, et al. Minimally invasive surgery alone compared with intensity-modulated radiotherapy for primary stage I nasopharyngeal carcinoma. Cancer Commun (London, England). 2019;39(1):75.

24. Li YZ, Xie CM, Wu YP, et al. Nasopharyngeal carcinoma patients with retropharyngeal lymph node metastases: a minimum axial diameter of $6 \mathrm{~mm}$ is a more accurate prognostic predictor than $5 \mathrm{~mm}$. AJR Am J Roentgenol. 2015;204(1):20-3.

25. Xiao W, Liu S, Tian Y, et al. Prognostic significance of tumor volume in locally recurrent nasopharyngeal carcinoma treated with salvage intensity-modulated radiotherapy. PloS One. 2015; 10(4):e0125351.

26. He YX, Wang Y, Cao PF, et al. Prognostic value and predictive threshold of tumor volume for patients with locally advanced nasopharyngeal carcinoma receiving intensity-modulated radiotherapy. Chin J Cancer. 2016;35(1):96.

27. Peng H, Chen L, Tang LL, et al. Significant value of (18)F-FDGPET/CT in diagnosing small cervical lymph node metastases in patients with nasopharyngeal carcinoma treated with intensitymodulated radiotherapy. Chin J Cancer. 2017;36(1):95.

28. Huang L, Chua MLK. Surgery as an alternative to radiotherapy in early-stage nasopharyngeal carcinoma: innovation at the expense of uncertainty. Cancer Commun (London, England). 2020; 40(2-3):119-121.

Cite this article as: Ding XI, Liu Y-P, Hua Y-J, Zou X, Wang Z-Q, Xie Y-L, Chen M-Y. Clinical advances in nasopharyngeal carcinoma surgery and a video demonstration. Visualized Cancer Medicine. 2021; 2, 2. 